\title{
Glucosyltyrosine in Silkworm Haemolymph as a Transient Metabolite of Insects
}

\author{
Minoru Isobe, Naoto Kondo, Kunio ImaI, Okitsugu Yamashita ${ }^{\dagger}$ and Toshio Goto \\ Laboratory of Organic Chemistry and ${ }^{\dagger}$ Laboratory of Sericultural Science, \\ Faculty of Agriculture, Nagoya University, \\ Chikusa, Nagoya 464, Japan
}

Received September 1, 1980

\begin{abstract}
Trehalose, the major haemolymph sugar in the silkworm, is partly regulated in concentration by diapause hormone. During the course of our re-investigation on the developmental changes of sugars in polyvoltine silkworm pupae, a new sugar (Sugar-A) was found out at the late stage of pupal-adult development. The amount of Sugar-A was five times as much as that of trehalose at its maximum, but it disappeared almost completely with emergence. The structure of Sugar-A was determined as $\beta$-glucosyl- $O$-tyrosine by $270 \mathrm{MHz}$ PMR, FDMS and chemical methods. The physiological function of glucosyltyrosine is also discussed.
\end{abstract}

Important phenomena in insects are under the control of the several organic compounds, as the hormones such as ecdysone, juvenile hormone, brain hormone, diapause hormone, etc. We have long been involved in the chemical studies on the diapause hormone $(\mathrm{DH}){ }^{11}$ and have also become interested in the dynamic or transient metabolites concerning the diapause. DH in the silkworm is secreted from the suboesophageal ganglion and induces the embryonic (egg) diapause. ${ }^{2)}$ DH also regulates the various metabolisms in developing ovaries for the preparation of their eggs to diapause. For instance, Yamashita (one of the authors) and Hasegawa ${ }^{3}$ disclosed that the ovary glycogen in the female silkworm was synthesized at the oocytes from glucose which derived from the blood (haemolymph) trehalose digested by the action of ovary trehalase. Enhanced activity of the trehalase by $\mathrm{DH}$ resulted in a rapid diminution of the blood trehalose. The trehalose content was determined in enzymatic method by a combination of trehalase and glucoseoxydase, which was a highly specific method. We herein describe our reinvestigated results about these carbohydrates and others in the haemolymph by a relatively nonspecific method, thus by means with HPLC and colorimetric or refractive-index detection. A new sugar (Sugar-A) was found in the silk- worm haemolymph as the major sugar component at a certain pupal stage, and the structure of Sugar-A was elucidated. We will also discuss the possible physiological function of this metabolite in the silkworm.

\section{METHODS AND RESULTS}

Materials. Haemolymph was collected from the pupae of the polyvoltine race of the silkworm $\left(\mathrm{N}_{4}\right)$ by cutting their final segments. The liquid was frozen rapidly to $-78^{\circ} \mathrm{C}$ and then kept at $-20^{\circ} \mathrm{C}$ until use. For the analyses on the developmental changes, four of the pupae were sacrificed every day throughout their pupal-adult development. Each aliquot of the haemolymph $(0.1 \mathrm{ml}$ out of the stored ca. $0.5 \mathrm{ml})$ was voltexed with ethanol $(0.4 \mathrm{ml})$ and centrifuged. The supernatant $(0.4 \mathrm{ml})$ was transferred into a vial, evaporated to dryness and then dissolved in $50 \mu \mathrm{l}$ of distilled water.

HPLC analyses. Each sample prepared above was analyzed by HPLC under the following condition: JASCO Tri-Rotar; Lichrosorb- $\mathrm{NH}_{2}$ (E. Merck), $2.5 \times$ $250 \mathrm{~mm}$; acetonitrile/water $=70 / 30,1.0 \mathrm{ml} / \mathrm{min} ; \mathrm{RI}$ detector (Shodex RI, SE-11). Ten microliter each of the sample was injected for analysis, and some of the typical chromatograms ( 1 and 6 day-old male pupae) are exemplified in Fig. 1. The fractions being positive to sugar test (anthron-sulfuric acid) are indicated by hatching in the figure. Change of these concentrations are summarized in Fig. 2.

Preparation of Sugar- $A$. Figures 1 and 2 suggested the presence of a new blood sugar (Sugar-A) in both 


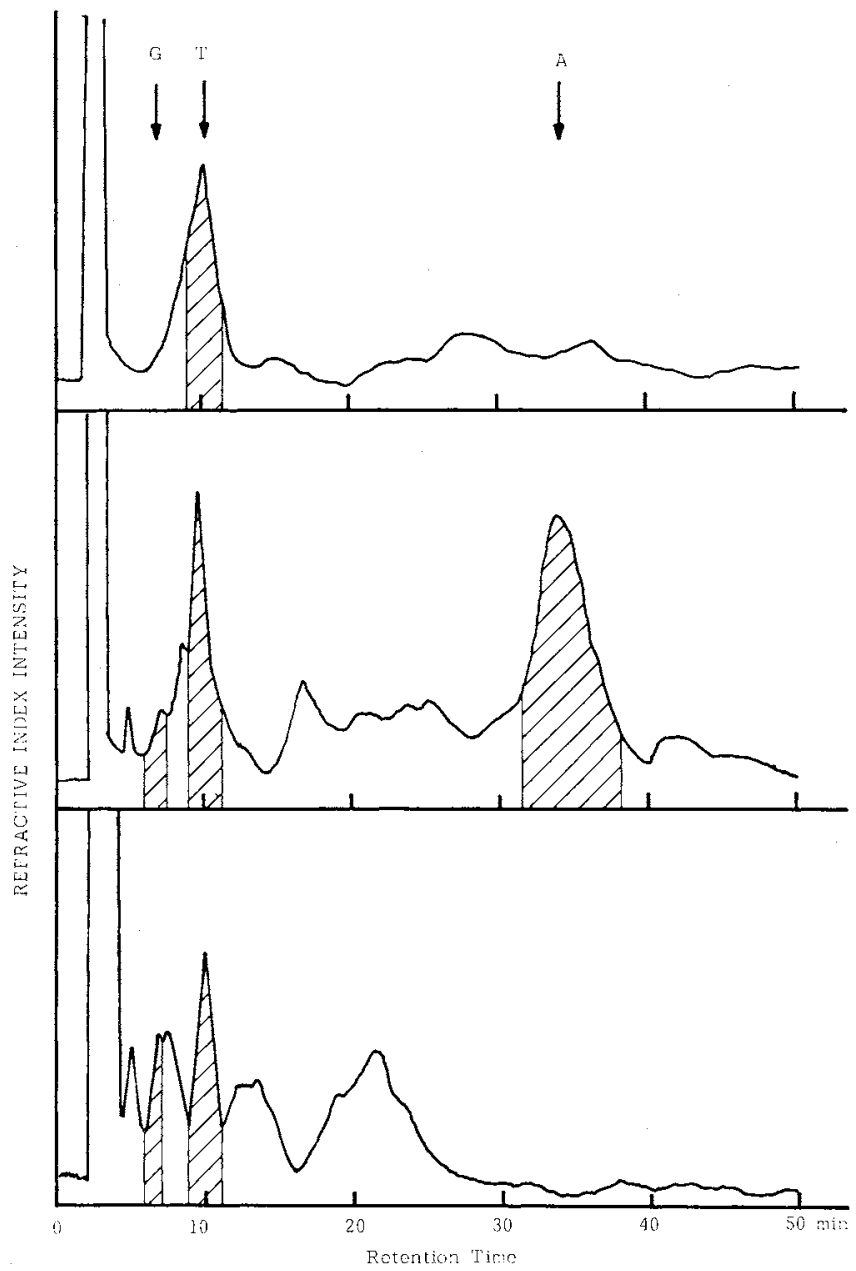

Fig. 1. Typical Chromatograms in Male Pupa of One-day Old [Bottom], Six-day Old [Middle] and One-day Old Adult [Upper] Columns, Respectively.

G, glucose; T, trehalose and A, Sugar-A (glucosyltyrosine).

sexes at the late pupal stage. In order to determine the chemical structure of Sugar-A, 40 six-day-old male pupae were sacrificed to collect $1 \mathrm{ml}$ of haemolymph. The haemolymph was mixed with $3 \mathrm{ml}$ of ethanol and centrifuged. The supernatant was evaporated to dryness, re-dissolved in $1 \mathrm{ml}$ of distilled water, and then filtered through teflon-wool. The clear solution was separated by HPLC under the similar condition, and the Sugar-A fraction was collected, which was purified twice (repeatedly) to afford white crude crystals. They were recrystallized by dissolving in water $(c a .0 .1 \mathrm{ml})$ and aliquot of ethanol at $c a .60^{\circ} \mathrm{C}$ and then by standing the solution at $5^{\circ} \mathrm{C}$ for one week to give colorless needles (mp $240^{\circ} \mathrm{C} ; 665 \mu \mathrm{g}$ ). The following data were obtained from the crystalline material of Sugar-A, which showed positive to anthron and also to ninhydrin tests.

\section{Characterization of Sugar $-A$}

NMR spectra. PMR spectrum $\left(270 \mathrm{MHz}\right.$ in $\left.\mathrm{D}_{2} \mathrm{O}\right)$ of Sugar-A showed the presence of p-disubstituted benzene $\left[\delta 7.22 \mathrm{ppm}\left(4 \mathrm{H}, \mathrm{A}_{2} \mathrm{X}_{2}\right)\right]$ and $-\mathrm{CH}_{2}-\mathrm{CH}-$ $[\delta 3.0 \sim 3.4 \mathrm{ppm}(2 \mathrm{H}, \mathrm{m}) ; \delta 3.98 \mathrm{ppm}(1 \mathrm{H}, \mathrm{dd}, J=5.0$, $8.0 \mathrm{~Hz})]$. The spectrum also indicated the signals corresponding to the following sugar-like moiety, thus anomeric proton $[\delta 5.16 \mathrm{ppm}(1 \mathrm{H}, \mathrm{d}, J=7.2 \mathrm{~Hz})]$ and six other protons $[\delta 3.5 \sim 3.9 \mathrm{ppm}(\mathrm{m})]$. Chemical shifts of CMR are listed in Table I, where the authentic numbers of tyrosine, glucose and galactose are also depicted for comparison. Figure 3 shows the PMR spectra with spin decoupling studies.

Mass spectra. The field desorption mass spectra (FDMS) were recorded on JEOL 01-SG-2 and D-300 


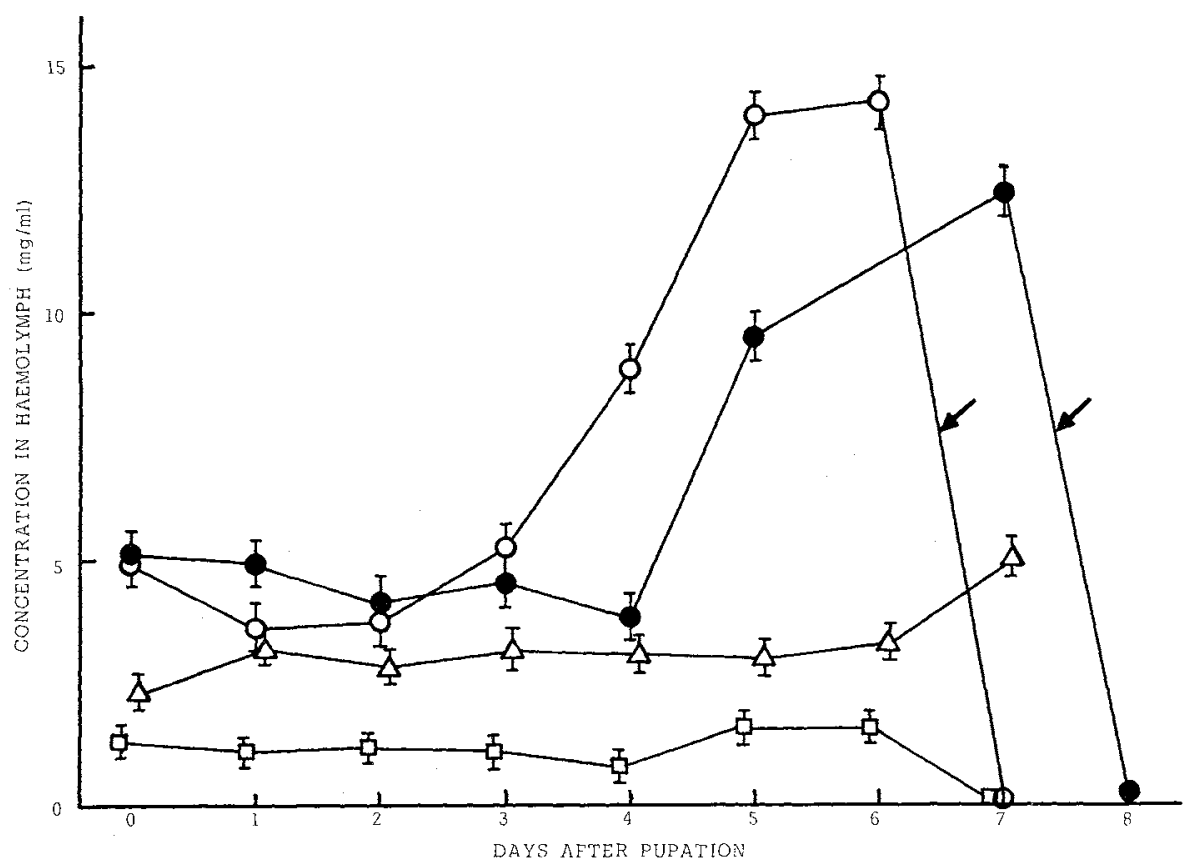

FrG. 2. Developmental Changes of Sugars in the Silkworm Haemolymph.

--o, Sugar-A (glc-tyr) in male; - - Sugar-A (glc-tyr) in female; $\square-\square$, trehalose in male and female; $\Delta-\Delta$, glucose in male and female; $\Rightarrow$, emergence.

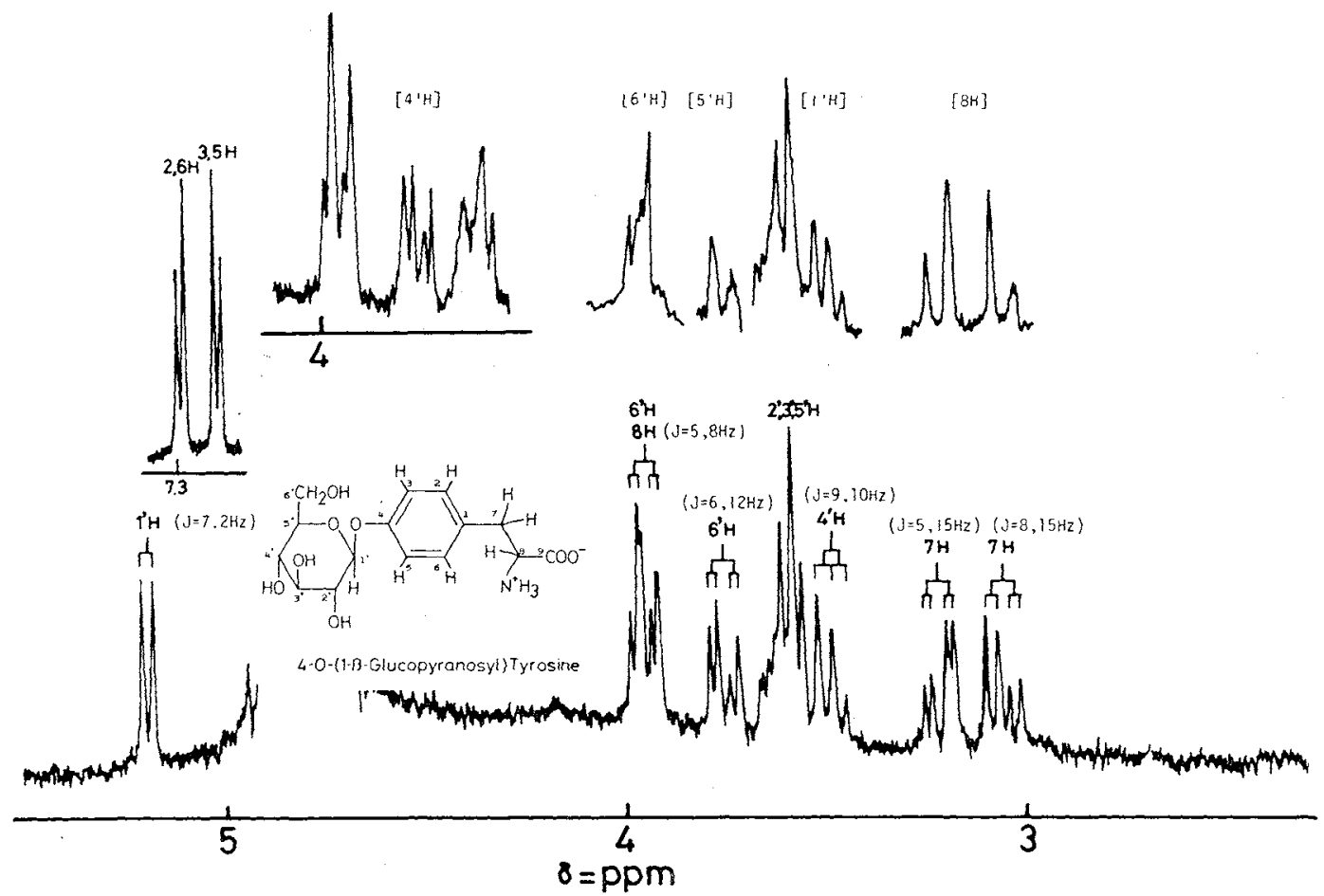

FIG. 3. $270 \mathrm{MHz}$ PMR Spectra of Sugar-A (Glucosyltyrosine) in $\mathrm{D}_{2} \mathrm{O}$.

Normal spectra in lower and middle lines. Spin-decoupled spectra in upper lines; [ ] indicating the irradiated protons. 

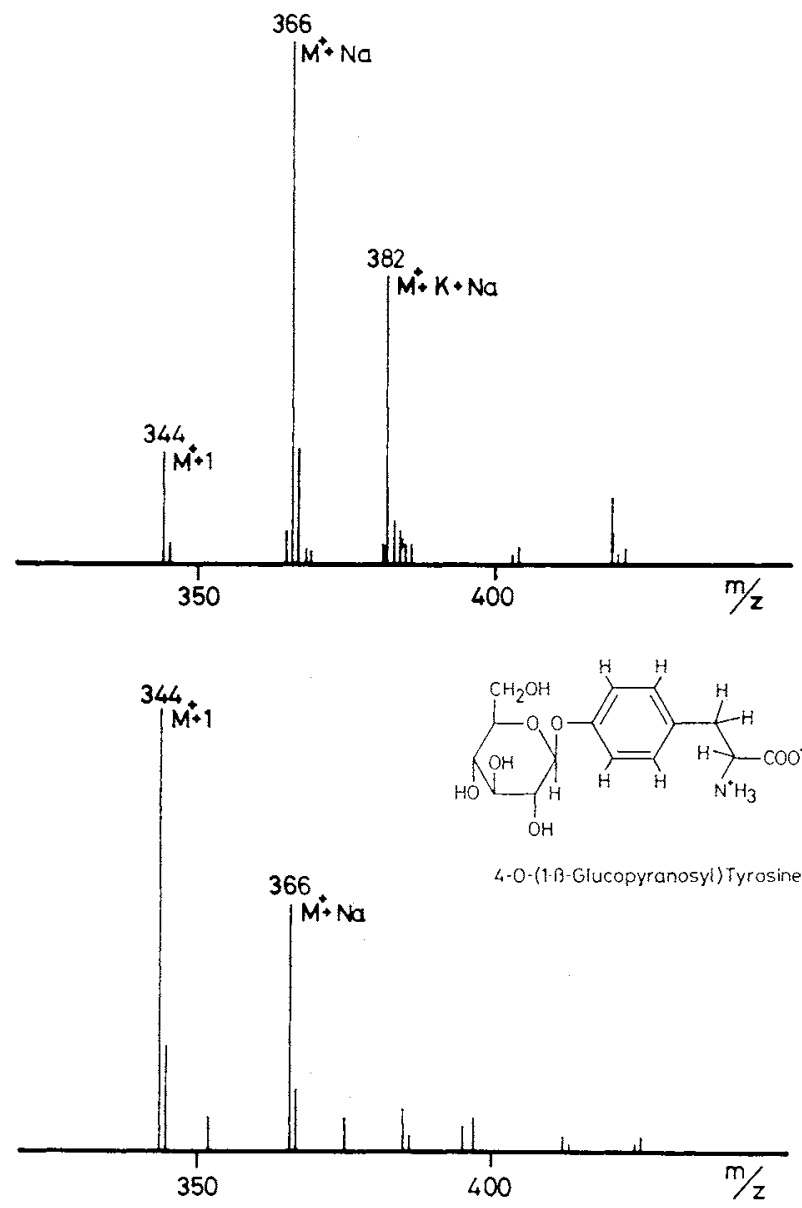

FIG. 4. Field Desorption Mass Spectra of Sugar-A (Glucosyltyrosine).

Emitter Current $18 \mathrm{~mA}$ (Lower), $22 \mathrm{~mA}$ (Upper).

instruments to show $m / z 344(\mathrm{M}+1), 366(\mathrm{M}+\mathrm{Na})$ and $382(\mathrm{M}+\mathrm{Na}+\mathrm{K})$ as shown in Fig. 4 .

Ultraviolet absorption spectra. The UV spectra (recorded with JASCO UVIDEC 505) of Sugar-A showed the presence of phenyl ether $\left[\lambda_{\max } 270 \mathrm{~nm}\right.$ $(\varepsilon=540) ; 276 \mathrm{~nm}$ (sh, $\varepsilon=480)$ at $\mathrm{pH}$ 's 1.7 and 11], since after the acid hydrolysis of Sugar-A (see the following section) the spectra changed $\left[\lambda_{\max } 272 \mathrm{~nm}\right.$, $280 \mathrm{~nm}(\mathrm{sh})$ at pH's 1 and $7 ; \lambda_{\max } 292 \mathrm{~nm}$ at pH 11]. Authentic tyrosine showed similar spectra $\left[\lambda_{\max } 273 \mathrm{~nm}\right.$, $280(\mathrm{sh})$ at $\mathrm{pH}$ 's 1 and $7 ; \lambda_{\max } 292 \mathrm{~nm}$ at $\left.\mathrm{pH} 11\right]$.

Acid hydrolysis. Sugar-A $(100 \mu \mathrm{g})$ was dissolved in $100 \mu 1$ of $2 \mathrm{~N} \mathrm{HCl}$ and heated in a vacuum-sealed tube at $100^{\circ} \mathrm{C}$ for $90 \mathrm{~min}$. The reaction mixture was evaporated to dryness with $\mathrm{N}_{2}$ stream, and the residue was dissolved in $20 \mu 1$ of distilled water. [1] One tenth of this hydrolysate was applied to an amino acid analyzer (JEOL JLC-6AH) to disclose the ninhydrinpositive constituent being tyrosine. Incidentally, Sugar-
A itself appeared at a similar retention time as that of proline. [2] The acid hydrolysate $(13.8 \mu 1)$ was analyzed by HPLC for the determination of its sugar constituent. Under the similar separation condition as was described before, the sugar appeared at the same retention time as that of glucose and galactose. The yields in the acid hydrolysis of Sugar-A were $34 \%$ for tyrosine and $30 \%$ for glucose.

\section{DISCUSSION}

Chemical structure of Sugar-A being 4-O-[ $\beta$ glucopyranosyl]tyrosine

The chemical structure of Sugar-A was determined as $4-O-[\beta$-glucosyl]tyrosine by the following reasons. Two constituents after the acid hydrolysis were identified by liquid chromatography to be glucose (or galactose) and also by amino acid analysis to be tyrosine. 
Table I. Chemical Shifts in CMR of Sugar-A AND Authentic TrRosine, Glucose aNd Galactose

\begin{tabular}{cccccc}
\hline $\begin{array}{l}\text { Carbon Observed Tyrosine } \\
\text { number }\end{array}{ }^{a}$ ppm & ppm $(A)^{b}$ & $\begin{array}{l}\text { Glucose } \\
\text { ppm }(\Delta)^{b}\end{array}$ & $\begin{array}{l}\text { Galactose } \\
\text { ppm }(\Delta)^{b}\end{array}$ \\
\hline 1 & - & 125.1 & & \\
2,6 & 131.8 & $132.0(0.2)$ & & \\
3,5 & 117.9 & $119.9(2.0)$ & & \\
4 & - & 166.0 & & \\
7 & 37.1 & $41.4(4.3)$ & & \\
8 & 54.8 & $59.3(4.5)$ & & \\
9 & - & 184.1 & & & \\
$1^{\prime}$ & 100.7 & & 96.7 & 97.3 \\
$2^{\prime}$ & 74.0 & & $74.9(0.9)$ & $72.9(-1.1)$ \\
$3^{\prime}$ & 77.2 & & $76.7(-0.5)$ & $73.7(-3.5)$ \\
$4^{\prime}$ & 70.5 & & $70.4(-0.1)$ & $69.6(-0.9)$ \\
$5^{\prime}$ & 76.6 & & $76.5(-0.1)$ & $75.9(-0.7)$ \\
$6^{\prime}$ & 61.6 & & $61.6(0.0)$ & $61.3(-0.3)$ \\
\hline
\end{tabular}

a Carbon number assignments of Sugar-A correspond to glucosyltyrosine.

$b$ Numbers in parentheses with authentic chemical shifts are the difference from the observed ones.

The molecular weight, $m / z 343$ (FDMS), of Sugar-A was consistent with the proposed structure. Bathochromic shift in the UV spectra in alkaline media was not observed on the Sugar-A, however, $20 \mathrm{~nm}$ shift was observed only on the acid hydrolysate; therefore it was disclosed that tyrosine linked at the phenolic oxygen with the sugar to form a glucosidic bond. The sugar constituent of Sugar-A was determined to be glucose by HPLC and NMR spectra; CMR data (see Table I) and also by the PMR $(270 \mathrm{MHz})$ spectra. In particular, the latter showed the presence of axial $\mathrm{C}-4^{\prime}-\mathrm{H} \quad[\delta 3.50 \mathrm{ppm}(\mathrm{t}, J=9,10 \mathrm{~Hz})]$ to eject the possibility of galactose. Glycosidic linkage was assigned by the chemical shifts of C-1' appearing at $\delta 100.7 \mathrm{ppm}$ and by the coupling constant of $\mathrm{H}-1^{\prime}$ being $7.2 \mathrm{~Hz}$ as axial orientation.

\section{Developmental changes in glucosyltyrosine in haemolymph}

The quantitative analyses on glucosyltyrosine (GLC-TYR) as well as trehalose and glucose were carried out in the haemolymph throughout the pupal-adult development of silkworms (Fig. 2). Those amounts were based on the RI (refractive index)-intensity of respective sugars at their retention times on HPLC. These values must be re-estimated by comparing the ones with other quantitative analyses.

As for free sugars, glucose and trehalose, their developmental changes were not inconsistent with the results obtained before by colorimetric analyses combined with enzymatical hydrolysis. $^{32)}$ GLC-TYR content in Fig. 2 was estimated from the calculation curve which was based on the crystalline GLC-TYR. The content stayed at about $4 \sim 5 \mathrm{mg} / \mathrm{ml}$ during the first half period, but abruptly enhanced at the middle phase of the pharate adult development. It reached to the maximum levels at the latest phase as much as ca. $15 \mathrm{mg} / \mathrm{ml}$; this amount being re-evaluated by the Amino Acid Analyzer which showed $16.0 \mathrm{mg} / \mathrm{ml}$ for the 6-day old male pupae. By the completion of the adult emergence, GLC-TYR decreased sharply to almost trace levels. For the females, the comparable profile of the developmental changes in these sugars and GLC-TYR was observed as shown in Fig. 2.

\section{Physiological roles on glucosyltyrosine}

Glucosyltyrosine (GLC-TYR) was found in the haemolymph of pharate adults of silkworms, and was first identified by chemical methods in this paper. The concentrations of GLC-TYR amounted to four to five times higher (Fig. 2) in molar than that of trehalose which has been believed to be a major blood sugar. The mode (appearance and disappearance) of this compound may be closely correlated to the developmental stages of silkworm; thus, at the middle stage of pharate adult development, the histolysis of larval tissues is almost completed and the histogenesis of adult tissues proceeds vigorously. However, any sexual dimorphism was not observed throughout the pharate adult development. Therefore, it is conceived for GLC-TYR to be a metabolite related to the adult formation not to the reproductive maturation.

Chen et $a l^{4)}$ have recently reported the first finding of a higher concentration of GLC-TYR in mature larvae of Drosophila busckii. Al- 
though they did not determine the concentration of GLC-TYR in their haemolymph, they disclosed from the data on whole larvae that this sugar-amino acid concerned the sclerotization process for puparium formation at larval-pupal moulting. Similarly in silkworms, GLC-TYR would be of importance as a tyrosine reservoir for cuticular formation of adults.

Tyrosine in GLC-TYR may be supplied largely by the breakdown of the Storage Protein-2 which comprises an abundance of tyrosine and phenylalanine during the histolysis in the silkworm. ${ }^{.5}$ The released amino acids will be reserved temporally in haemolymph until the protein synthesis for adults (at normal tyrosine levels) will begin. For an eventual accumulation in haemolymph of tyrosine, its conjugation with glucose could be facilitated to keep the poorly soluble tyrosine in solution and to avoid its decomposition by oxidation with the phenoloxydases present in the haemolymph..$^{6)}$ Glycogen in fat body, on the other hand, is simultaneously broken down to form glucose convertible into t:ehalose, ${ }^{7 /}$ while some of the glucose formed will be used for the conjugation of tyrosine. These facts imply that the conjugate should temporally reserve the amount of tyrosine as well as glucose in haemolymph for utilization in the later development, cross linking of the proteins in the cuticular formation.

We also found GLC-TYR in the larval haemolymph of the armyworm, Leucania separata (Lepidoptera), and did in the univoltine silkworm pupae, but failed in the silkworm larvae. In most of the dipteran, tyrosine- $O$-phosphate, ${ }^{8)} \quad \beta$-alanyltyrosine ${ }^{9)}$ or $\gamma$-glutamylphenylalanine ${ }^{10}$ are known to be involved in their sclerotization to serve tyrosine. And thus, $\beta$-glucosyl- $O$-tyrosine in the silkworm haemolymph should be recognized as the transient metabolite for cuticular formation.

Further studies on the dynamic or transient metabolites in the silkworm are in progress.

Acknowledgments. This work was partly supported by the Grant-in-Aid for Scientific Research from the Ministry of Education, Science and Culture of Japan. Authors are grateful to Professor Miyazawa and Dr. Yokoyama at Tokyo University for $270 \mathrm{MHz}$ PMR rneasurements.

\section{REFERENCES}

1) (a) M. Isobe, K. Hasegawa and T. Goto; J. Insect Physiol., 19, 1221 (1973); (b) I. Kubota, M. Isobe, K. Imai, T. Goto, O. Yamashita and K. Hasegawa, Agric. Biol. Chem., 43, 1075 (1979) and the references cited therein.

2) (a) K. Hasegawa, Proc. Jpn. Acad., 27, 667 (1951); (b) S. Fukuda, ibid., 27, 672 (1951); (c) K. Hasegawa, Nature, 179, 1300 (1957).

3) (a) O. Yamashita and K. Hasegawa, J. Sericult. Sci., 34, 235 (1965) (in Japanese); (b) idem., Proc. Jpn. Acad., 43, 547 (1967); (c) idem, J. Insect Physiol., 16, 2377 (1970).

4) P. S. Chen, H. K. Mitchell and M. Neuweg, Insect Biochem., 8, 279 (1978).

5) S. Tojo, M. Nagata and M. Kobayashi, Insect Biochem., 10, 289 (1980).

6) T. Hashiguchi, J. Sericult. Sci., 39, 37 (1970) (in Japanese).

7) O. Yamashita and K. Hasegawa, J. Insect Physiol., 20, 1749 (1974).

8) K. D. Lunan and H. K. Mitchell, Arch. Biochem. Biophys., 132, 450 (1969).

9) L. Levenbook et al., Biochem. J., 113, 837 (1969).

10) R. P. Bodnaryk, Comp. Biochem. Physiol., 43B, 587 (1972). 